\title{
Editorial: bio- and chemo-mechanical processes in geotechnical engineering
}

The rapidly expanding field of coupled multi-physics processes is applicable to a number of geotechnical engineering situations. Generally, the various evolving physical phenomena interact with each other and increase the complexity of the engineering problems. The evolution of these phenomena must be examined over significant periods of time, especially when issues of durability are concerned.

Some of these aspects have been presented in previous Géotechnique symposia in print: suction and non-saturation aspects in 1983, 2003 and 2011; non-isothermal conditions in 2009. This 17th edition of the biennial Géotechnique symposium in print series extends the area of multi-physical analysis to consider the evolution of the mechanical behaviour of soils and rocks exposed to biological and chemical processes.

In recent years, substantial advances have been made in understanding the coupling between chemical and biological processes and mechanical and hydraulic behaviour in soils and rocks. At the same time, experimentation and modelling capabilities have progressed significantly, allowing effective design of geotechnical applications. The need for such analyses arises (for example) in chemical and biological soil improvement; nuclear, hazardous and municipal waste containment; petroleum and natural gas extraction; methane hydrate exploitation; carbon dioxide sequestration; and the assessment of pavement durability.

In such areas, instances of complexity and interaction are many, mainly because of the coexistence of several constituents and phases, their interactions, their reactivity, and their often non-linear behaviour. Flow involves water and gas, including the transport of chemical species and bacteria. Geomaterial deformation depends not only on classical effective stress, suction and temperature, but also on the history of chemical and bacterial activity in the material. Experimental observations are often difficult to carry out, and laboratory and in situ tests are costly challenges. An understanding of the material behaviour to be observed requires the control or measurement of many different parameters. Modelling inevitably implies numerical analyses. Coupled transient analyses are in fact a characteristic feature of this field; proper variables must be selected which can describe the behaviour of the geomaterials subjected to chemical and biological phenomena. Robust numerical techniques are required in order to solve, with sufficient accuracy, the strongly coupled analytical systems. Hence, progress in coupled bio- and chemo-mechanical processes in geotechnical engineering requires advances in theoretical formulations, numerical analyses, constitutive modelling and laboratory techniques, as well as detailed examination of well-documented field cases.

The response to this symposium in print was substantial, with over 91 abstracts offered by authors throughout the world, reflecting the interest and importance of this topic. The panel invited 48 among them to be presented as fulllength papers. Following the standard review process of Géotechnique, 14 papers have so far been accepted for publication; they are published in two thematic issues, this issue and the following issue of the journal. In these two thematic issues we cover some of the variety of approaches and techniques that have recently developed in this area. The first issue contains seven papers; emphasis is given to the presentation of the chemo-mechanical interactions in multiphase geomaterials. The second issue compiles seven papers dealing with both bio- and chemo-mechanical couplings.

Due to the strict deadlines that had to be set during peerreviewing, some articles were not ready for publication according to the planned schedule. They were moved to the regular publication process of the journal. I am continuing to handle their review and acceptance stages.

The two thematic issues will be accompanied by a fullday symposium, to be held on 3rd June 2013 at the Institution of Civil Engineers (ICE) in London, to take stock of different approaches to the experimental characterisation, constitutive modelling and design/monitoring of geotechnical engineering applications. We plan to have three eminent keynote addresses to provide the context of the papers. The papers themselves will be summarised by session chairpersons in three thematic sessions, corresponding to the three sub-themes of the symposium (bio-mechanical processes, chemo-mechanical processes, field applications); some of them will be presented by the authors and there will be substantial time available for discussion by the audience. In particular, many of the authors will be present enabling a valuable depth of discussion. I believe that the symposium will provide an excellent tool for discussion, learning and outlining future developments in this fascinating and critically important area. The meeting is open to all interested people and those wishing to take part in it are invited to register by visiting http://www.ice.org.uk/geosymp. The participants in the symposium are encouraged to read the papers before attending the symposium meeting.

It was my great pleasure to act as the chairman of the Géotechnique advisory panel sub-committee and to have been the guest editor of this and the next issue of Géotechnique, which contain the symposium in print papers. Also, it will be my honour to chair the symposium in June. ICE, ICE Publishing and the members of the sub-committee are working to make the meeting as productive and lively as possible. We also intend to have technical poster presentations during the breaks to inspire further ideas.

I wish to thank the members of the symposium subcommittee for their enormous amount of work. I am extremely grateful to all the reviewers of the symposium papers who were very helpful in completing their reviews to a very tight time scale. I would like also to extend my thanks to Miss Sarah Walker, journals editorial coordinator at ICE Publishing, who efficiently helped me in the organisation of the activities.

Lyesse Laloui

Ecole Polytechnique Fédérale de Lausanne

Symposium in print sub-committee chairman

Professor Lyesse Laloui, Ecole Polytechnique Fédérale de Lausanne, Switzerland

Symposium in print sub-committee members

Dr Malek Bouazza, Monash University, Australia

Dr Peter Cleall, Cardiff University, UK 
Professor Yu-Jun Cui, ENPC, France

Dr Alessio Ferrari, EPFL, Switzerland

Professor Stephanie Glendinning, Newcastle University, UK

Professor Kenichi Soga, University of Cambridge, UK
Professor Alessandro Tarantino, University of Strathclyde, UK Professor David Toll, Durham University, UK

Dr Antonis Zervos, Southampton University, UK Professor Hehua Zhu, Tongji University, China 